\title{
Erratum to: Interaction between groundwater, the hyporheic zone and a Chalk stream: a case study from the River Lambourn, UK
}

David J. Allen • W. George Darling •

Daren C. Gooddy • Daniel J. Lapworth •

Andrew J. Newell • Ann T. Williams • Debbie Allen •

Corinna Abesser

Erratum to: Hydrogeology Journal (2010)

DOI 10.1007/s10040-010-0592-2

On the front page, the article has been incorrectly assigned as '(C) British Crown Copyright 2010'. It should be 'British Geological Survey (C) NERC 2010'.

Published online: 13 July 2010

British Geological Survey C NERC 2010

The online version of the original article can be found at http://dx. doi.org/10.1007/s10040-010-0592-2.

D. J. Allen $(\cdot) \cdot$ W. G. Darling • D. C. Gooddy • D. J. Lapworth •

A. J. Newell $\cdot$ A. T. Williams $\cdot$ D. Allen $\cdot$ C. Abesser

British Geological Survey,

Maclean building, Crowmarsh Gifford, Wallingford,

Oxon OX10 8BB, UK

e-mail: dja@bgs.ac.uk

Tel.: +44-1491-692305

Fax: +44-1491-692345 